\title{
Clinical Study \\ Calcific Uremic Arteriolopathy on Multimodal Combination Therapy: Still Unmet Goal
}

\author{
Usman Hammawa Malabu, ${ }^{1}$ Valli Manickam, ${ }^{2}$ George Kan, ${ }^{2}$ Susan Lynette Doherty, ${ }^{3}$ \\ and Kunwarjit Singh Sangla ${ }^{1}$
}

\footnotetext{
${ }^{1}$ Department of Endocrinology, The Townsville Hospital, 100 Angus Smith Drive, Douglas, QLD 4814, Australia

${ }^{2}$ Renal Medicine, The Townsville Hospital, 100 Angus Smith Drive, Douglas, QLD 4814, Australia

${ }^{3}$ Occupational Therapy, The Townsville Hospital, 100 Angus Smith Drive, Douglas, QLD 4814, Australia
}

Correspondence should be addressed to Usman Hammawa Malabu, umalabu@yahoo.com

Received 1 September 2011; Revised 18 November 2011; Accepted 3 December 2011

Academic Editor: R. Khanna

Copyright (C) 2012 Usman Hammawa Malabu et al. This is an open access article distributed under the Creative Commons Attribution License, which permits unrestricted use, distribution, and reproduction in any medium, provided the original work is properly cited.

\begin{abstract}
Background. Calcific uremic arteriolopathy (CUA) or calciphylaxis though generally noted for its high mortality, recent case reports have shown promising results using single agent therapies. However, it is not clear whether combination therapeutic agents will improve course of the disease. Objective. To determine clinical outcome in subjects with CUA on multimodal treatment. Methods. All patients with end-stage renal failure (ESRF) at The Townsville Hospital, Australia, from April 1, 2006, to March 31, 2011, with diagnosis of CUA were retrospectively studied. Results. Six subjects with CUA (4 females and 2 males) were on various combination therapeutic agents comprising sodium thiosulphate, hyperbaric oxygen, prednisolone, cinacalcet, and parathyroidectomy in addition to intensified haemodialysis, specialist local wound care, and antibiotics. The wounds failed to heal in 3 patients while 5 of the 6 subjects died; cause of death being sepsis in 3 and myocardial infarction in 2. Conclusion. Prognosis of CUA remains poor in spite of multimodal combination therapy. Further prospective studies on a larger population are needed to verify our findings.
\end{abstract}

\section{Introduction}

Calcific uremic arteriolopathy (CUA) or calciphylaxis is a syndrome of painful skin necrosis and vascular calcification with high morbidity and mortality. It occurs primarily in patients with end-stage renal disease $[1,2]$. Though generally a rare syndrome, it is increasingly described in subjects undergoing renal dialysis. Calciphylaxis has also been described in subjects with normal renal function including primary and secondary hyperparathyroidism [3, 4]. A common factor linking non-renal-failure-related presentation of the same disease is elevated parathyroid hormone with or without elevated calcium-phosphate product leading to calcification of small vessels. The consequences of these are significant mortality of $80 \%$ principally from multiple end organ damage due to ischemia and infarction commonly complicated by infection; sepsis being the principal cause of death [1-3]. Thus, traditional care addresses the calcium-phosphate-PTH axis: substituting calcium with non-calcium as phosphate binders, strict dietary phosphate control, cautious vitamin D analogs, calcimimetics, and surgical parathyroidectomy if necessary. Newer approaches focus on intravascular and tissue mineralization: dissolution of calcium deposits with the use of sodium thiosulfate, altering osteoprotegerin, and NF- $\kappa$ B pathways with bisphosphonates $[4,5]$. Recently, single agent treatment such as sodium thiosulphate and hyperbaric oxygen has yielded various successes in care of calciphylaxis [5-7]. Furthermore, favourable outcome was recorded in limited reports on CUA using cinacalcet or parathyroidectomy to achieve targeted normalised calcium, phosphate, and parathyroid hormone levels $[8,9]$. Yet usefulness of these agents in the form of combination therapy has not been fully explored $[10,11]$. The aim of the study was to review all cases of CUA seen in the last five years and determine its clinical course in the setting of multimodality care. 


\section{Material and Methods}

All patients diagnosed to have CUA at The Townsville Hospital, Australia, from April 1, 2006, to March 31, 2011, were retrospectively studied. Subjects with nonuremic calciphylaxis were excluded from the study. Approval for the study was sought from the hospital's ethical committee. Data collected included age, gender, onset of dialysis to diagnosis of calciphylaxis, duration of the skin lesion, and outcome defined as completely or partially healed skin lesions or death of a subject. In all subjects, calciphylaxis trigger agent(s) were ceased-calcium-based phosphate binders and alphacalcidol; none was on warfarin. All the patients were on oral erythropoietin for anemia in addition to iron infusion in 2 patients. Primary cause of the renal failure was also determined as well as comorbidities. Biochemical profile of subjects with CUA at diagnosis and within 6 months on multimodal care was recorded. These included corrected calcium, phosphate, albumin, calculated calcium-phosphate product, and parathyroid hormone.

The patients included in this study were reviewed by the vascular team, and a second opinion was obtained from a dermatologist. All lesions had a punch biopsy performed across the ulcer edge, but if the specimen was insufficient to demonstrate calciphylaxis, then a wedged biopsy was performed to confirm the diagnosis. All biopsies were analysed by the same pathologist; the diagnostic criteria used were medial calcification of dermal small and medium-sized arterioles and arteries, intimal fibroblastic and endothelial proliferation associated with ischemic necrosis of subcutaneous adipose tissue, and epidermal ulceration. Mediumsized arteries with medial calcific sclerosis (Monckeberg's medial calcific sclerosis) with no intimal or endothelial proliferation were excluded from the study.

The patients' wounds were managed by clinical occupational and nurse therapist with specialist interest in wound healing and compression therapy. The dry necrotic lesions were gently hydrated to promote a moist wound environment, encouraging autolytic debridement and cell migration. An atraumatic dressing (silicone coated, Mepitel) and nonadherent absorbent pad were applied as secondary dressings. After autolytic debridement, a conformable silver polymembrane was applied and retained with light tubular support bandage. If the wound was infected, antibiotics were administered based on microscopy, culture, and sensitivity results following deep wound swab and blood cultures with input from the infectious disease specialist. To improve wound healing, daily hyperbaric oxygen was used in some patients. Each treatment lasted 90 minutes undertaken at 2.4 atmospheres absolute (ATA).

Methods used in lowering calcium-phosphate product and parathyroid hormone included emergency parathyroidectomy, use of non-calcium phosphate binderssevelamer $4.5 \mathrm{~g} /$ day-and cinacalcet $30 \mathrm{mg}$ daily. The latter being calcimimetic lowers parathyroid hormone by increasing sensitivity of the calcium receptor. All the patients had low calcium containing dialysate (calcium concentration $1.25 \mathrm{mEq} / \mathrm{L}$ ) and the dialysis-dose was increased from $8 \mathrm{~L}$ dialysate/day to $12 \mathrm{~L} /$ day. Oral prednisolone $50 \mathrm{mg}$ once daily was administered in 2 patients due to contraindication to parathyroidectomy and refusal to use sodium thiosulphate (STS). Twenty-five grams of STS $(100 \mathrm{~mL}$ of a solution at $25 \%$ STS) were infused three times per week in 3 patients for 1 to 3 months. The thiosulphate was administered immediately after dialysis. There were no signs, symptoms, or electrocardiographic evidence of hypocalcaemia at any time during the course of STS therapy. There was no discernible effect of each thiosulphate infusion on any of the plasma chemistry values or any other adverse effects such as metabolic acidosis or haemorrhagic complications. Wound response to the treatments was monitored clinically and by serial photography comparing before and after progress. In addition, patients were followed up in outpatient clinics to determine if the healed skin lesion relapsed or to determine cause of death had the patient died. Treatment for comorbidities was provided and followed up by the appropriate subspecialties.

Once discharged from the hospital, patients were followed up weekly by the clinical occupational therapist and by nephrologists 3 times a week at the renal dialysis session looking for relapse of the calciphylaxis. Results were presented in tabular form for all the patients. Comparison of biochemical profile was made at and within 6 months after the diagnosis of CUA with overall results depicted as mean \pm standard deviation, where applicable.

\section{Results}

Over the five-year period, we recorded 6 cases of CUA out of 201 subjects who had regular haemodialysis sessions at the unit as shown in Table 1 . Of the 6 subjects, two-third was females. Mean duration of dialysis was 20 months range 1 to 3 years. Infection was demonstrable in all subjects as revealed by wound swab microscopy, culture, and sensitivity. The commonest organism isolated was Pseudomonas aeruginosa in 4 subjects, namely, patient $1,4,5$, and 6 . Patient 6 in addition had methicillin resistant Staphylococcus aureus (MRSA). Patient 2 and 3 had MRSA and Klebsiella pneumonia, respectively. Modalities of treatment of the calciphylaxis apart from wound care and administration of antibiotics in all included cinacalcet in patient 1,3 , and 6; prednisolone was used in patient 3 and 4, while parathyroidectomy was emergently done in patient 2 and 3 with marked hyperparathyroidism and progressive disease despite medical therapy. Following the parathyroid surgery, whereas patient 2 had her wounds completely healed and remained in remission for 32 months, patient 3 did not respond to the parathyroidectomy and died 5 months later of sepsis. Below knee amputation was performed in only one subject, patient 5, who did not respond to local wound care, antibiotics, hyperbaric oxygen therapy, and administration of sodium thiosulphate. The CUA reoccurred rapidly within one week of the surgery which was complicated by sepsis following withdrawal of further treatment including haemodialysis. He died one month after the knee amputation. Side effects of the treatments offered were assessed, but none of the patients developed any untoward effects to prednisolone, cinacalcet, STS, or hyperbaric oxygen therapy. Overall, combination therapy for 
TABLe 1: Patients' characteristics and clinical course in subjects with end-stage renal failure presenting with calciphylaxis.

\begin{tabular}{|c|c|c|c|c|c|c|}
\hline Subject & Patient 1 & Patient 2 & Patient 3 & Patient 4 & Patient 5 & Patient 6 \\
\hline Age (years) & 48 & 53 & 63 & 72 & 74 & 86 \\
\hline Gender & Female & Female & Female & Female & Male & Male \\
\hline Cause of ESRF & Diabetes & Chronic GN & Diabetes & Diabetes & Diabetes & Obstructive uropathy \\
\hline $\begin{array}{l}\text { Duration RRT } \\
\text { (months) }\end{array}$ & 12 & 36 & 24 & 21 & 15 & 12 \\
\hline Site of lesion & Extensive* $^{*}$ & Distal & Distal & Extensive & Distal & Distal \\
\hline Microorganism & Pseudomonas & MRSA & Klebsiella & Pseudomonas & Pseudomonas & MRSA+pseudomonas \\
\hline Mode of therapy** & Cinacalcet STS & PTHX HBO & $\begin{array}{l}\text { PTHX HBO STS } \\
\text { prednisolone }\end{array}$ & $\begin{array}{l}\text { Cinacalcet } \\
\text { prednisolone }\end{array}$ & $\begin{array}{l}\text { STS HBO } \\
\text { amputation }\end{array}$ & Cinacalcet HBO \\
\hline Outcome & Dead & Dead & Dead & Dead & Dead & Alive \\
\hline Cause of death & Sepsis & $\begin{array}{l}\text { Myocardial } \\
\text { infarction }\end{array}$ & Sepsis & $\begin{array}{l}\text { Myocardial } \\
\text { infarction }\end{array}$ & Sepsis & Not applicable \\
\hline Comorbidities & $\begin{array}{l}\text { DM ESRF IHD } \\
\text { HTN Asthma }\end{array}$ & Epilepsy ESRF gout & DM ESRF gout & $\begin{array}{l}\text { IHD DM ESRF } \\
\text { MGUS }\end{array}$ & $\begin{array}{l}\text { DM ESRF } \\
\text { HTN arthritis }\end{array}$ & ESRF \\
\hline Skin lesion outcome & Failed & Healed & Failed & Healed & Failed & Healed \\
\hline Survival (months) & 1 & 32 & 5 & 4 & 4 & Alive \\
\hline
\end{tabular}

ESRF: end-stage renal failure, GN: glomerulonephritis, RRT: renal replacement therapy, *: extensive skin lesions involving the lower limbs, genitalia, abdominal wall, and breast, MRSA: methicillin resistant Staph aureus, ${ }^{* *}$ : mode of therapy apart from general wound care and antibiotics, STS: sodium thiosulphate, PTHX: parathyroidectomy, HBO: hyperbaric oxygen, IHD: ischemic heart disease, HTN: hypertension, MGUS: monoclonal gammopathy of unknown significance.

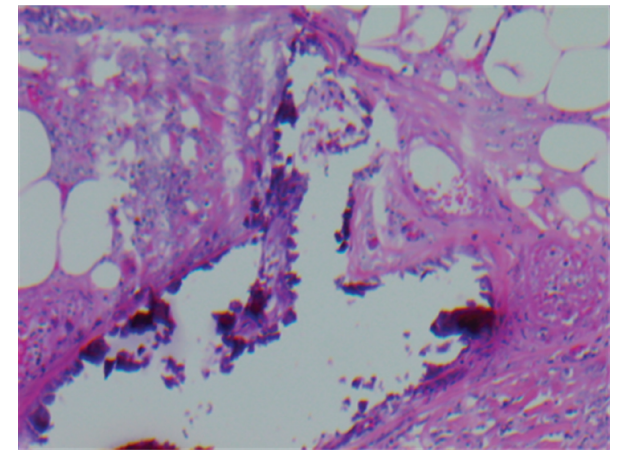

FIGURE 1: Histology of skin biopsy of patient 4 showing calcific arteriolopathy typical of calciphylaxis. Haematoxylin and eosin stain, magnification 200x.

CUA was offered in the form of dual in 4 subjects, triple and quadruple in one subject each as detailed in Table 3.

In addition to ESRF, other diseases were recorded premorbidly. These include ischemic heart disease in subject 1 and 4 though patient 2 and 4 both died suddenly, and autopsy confirmed myocardial infarction as a cause of death. Other comorbidities apart from diabetes in all but 2 patients, hypertension occurred in subject 1 and 5. Interestingly, patient 2 and 3 were on treatment for gout. Closer look at the survival details revealed only one patient is alive, the rest died mainly within 9 months of diagnosis of CUA.

The histology of the skin biopsy of the patients was analysed histologically as depicted in Figure 1. The defining histological characteristic found in all the cases was extensive calcification of small and medium-sized vessels of the dermis and subcutis layer with typical calcific thrombogenic microangiopathy. The deposition of calcium in the

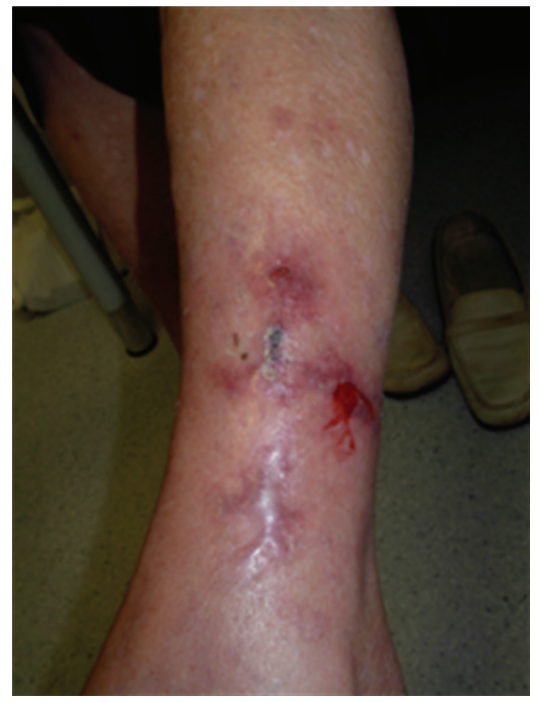

FIGURE 2: Successfully healed calciphylaxis following autolytic debridement and dressings along with treatment with cinacalcet and prednisolone in patient 4 who later died of myocardial infarction.

media was either segmental or circumferential and was accompanied by intimal hyperplasia, fibrosis, and thickening with smooth muscle fibre atrophy. Table 2 shows mean biochemical profile of subjects with CUA at diagnosis and within 6 months after. Calcium-phosphate product was similar in both groups, while serum parathyroid hormone was significantly lower after the diagnosis.

Figure 2 shows completely healed ulcers of calciphylaxis in patient 4 who had been on treatment for ischemic heart disease. She presented initially with extremely painful leg 
TABLE 2: Comparison of biochemical profile of subjects with calciphylaxis at diagnosis and within 6 months after the diagnosis.

\begin{tabular}{lccc}
\hline Test & At diagnosis & 3 to 6 months after diagnosis & \\
\hline Calcium $(\mathrm{mmol} / \mathrm{L})$ & $2.3 \pm 0.2$ & $2.4 \pm 0.2$ & value \\
Phosphate $(\mathrm{mmol} / \mathrm{L})$ & $2.0 \pm 0.8$ & $1.8 \pm 1.0$ & NS \\
Ca $\times$ P $(\mathrm{mmol} / \mathrm{L})$ & $4.5 \pm 1.0$ & $4.2 \pm 1.8$ & NS \\
PTH $(\mathrm{pmol} / \mathrm{L})$ & $80 \pm 53$ & $21 \pm 17$ & $<0.05$ \\
Albumin $(\mathrm{g} / \mathrm{L})$ & $28 \pm 7$ & $26 \pm 9$ & $\mathrm{NS}$ \\
\hline
\end{tabular}

SD: standard deviation, $\mathrm{Ca} \times \mathrm{P}$ : calcium-phosphate product, PTH: parathyroid hormone, NS: not significant.

TABLE 3: Multimodal care and outcome in subjects with calcific uremic arteriolopathy.

\begin{tabular}{|c|c|c|c|c|c|c|c|}
\hline Patient & Antibiotics & Sodium Thiosulfate & Cinacalcet & Hyperbaric Oxygen & Prednisolone & Parathyroidectomy & Outcome \\
\hline 1 & $\sqrt{ }$ & $\sqrt{ }$ & $\sqrt{ }$ & & & & Dead \\
\hline 2 & $\sqrt{ }$ & & & $\sqrt{ }$ & & $\sqrt{ }$ & Dead \\
\hline 3 & $\sqrt{ }$ & $\sqrt{ }$ & & $\sqrt{ }$ & $\sqrt{ }$ & $\sqrt{ }$ & Dead \\
\hline 4 & $\sqrt{ }$ & & $\sqrt{ }$ & & $\sqrt{ }$ & & Dead \\
\hline 5 & $\sqrt{ }$ & $\sqrt{ }$ & & $\sqrt{ }$ & & & Dead \\
\hline 6 & $\sqrt{ }$ & & $\sqrt{ }$ & $\sqrt{ }$ & & & Alive \\
\hline
\end{tabular}

ulcers with features of cardiac failure. Further echocardiographic assessment revealed extensive coronary calcification involving 3 main vessels. She was declared not for coronary bypass grafting and opted for palliative care. She was on the following oral agents: aspirin $100 \mathrm{mg}$, clopidogrel $75 \mathrm{mg}$, nicorandil $20 \mathrm{mg}$, isosorbide mononitrate $120 \mathrm{mg}$, frusemide $40 \mathrm{mg}$, atenolol $50 \mathrm{mg}$, candesartan-hydrochlorothiazide $16 / 12.5 \mathrm{mg}$, atorvastatin $40 \mathrm{mg}$, and amlodipine $10 \mathrm{mg}$ all were given once daily. Daily wound care and oral cinacalcet along with prednisolone were commenced to which she responded dramatically within one month and the lesions completely healed by second month as shown in Figure 2. She remained symptomatic of cardiac angina despite on multiple medications. Sadly, she died suddenly and autopsy confirmed myocardial infarction as the cause of death. On the other hand, patient 1 had painful bilateral legs lesions which progressed rapidly within a month with involvement of genitalia and breast. Sodium thiosulphate infusion and cinacalcet were given for one month without improvement. The patient declined further care and died of sepsis a week later following discontinuance of active treatment including haemodialysis.

All the patients had ESRF on haemodialysis; two-third due to diabetes. Other causes of ESRF recorded include chronic glomerulonephritis and obstructive uropathy in one subject each, respectively. All the subjects had lower limb skin lesions. In addition, both patients 1 and 5 had proximal involvement. The skin lesion partially healed in one subject and failed to heal in the 3 who died of overwhelming sepsis. On the other hand, the 2 subjects with successfully healed wounds died of myocardial infarction 1 to 2 years later.

\section{Discussion}

We have demonstrated poor outcome of calciphylaxis in subjects on multimodal combination therapies. All except one patient died within an average of 9 months from the diagnosis of the skin lesion; in line with others observation
$[1,2]$. Recently, reports have shown high survival rate and complete wound healing with the use of single agent therapy including sodium thiosulphate, hyperbaric oxygen, cinacalcet, and parathyroidectomy [5-9]. Surprisingly, such benefit was not evident in our subjects on multiagent treatment in the form of dual, triple, or quadruple therapies, similar to Kyritsis et al. observation [10], but in contrast to better outcome noted by Arenas et al. and by Baldwin and colleagues $[11,12]$.

It is interesting to note that though single or multipleagent therapies aimed at lowering calcium-phosphate product have shown some positive results in treatment of calciphylaxis, to-date no standard management is universally accepted to treat this condition. Nonetheless, noncalcium-containing phosphate binders, bisphosphonates, the calcimimetic cinacalcet, and vitamin $\mathrm{D}$ analogs have been used with this intent [13]. For instance, pamidronate, a bisphosphonate used singly or in combination with other agents, has been reported to respond favourably in subjects with calciphylaxis [4, 14]. Similarly, by lowering serum parathyroid hormone, cinacalcet has also proved to be beneficial in care of CUA [15]. Furthermore, emergency parathyroidectomy has been used in the treatment of patients with CUA associated with hyperparathyroidism refractory to medical therapy; however, case series have shown variable results $[1,9,16]$. Other treatment modalities include hyperbaric oxygen therapy which has been found useful in management of CUA by improving tissue oxygenation and promoting wound healing [7, 17]. Similarly, sodium thiosulfate increases solubility of calcium deposits in CUA with enhanced wound healing [6]. The success of sodium thiosulfate therapy alone or in combination with other agents in patients with calciphylaxis has been described in several case reports and case series $[5,18,19]$. Despite the observation by others of favourable outcome using multiple agents in care of CUA, we recorded high mortality even after successful wound healing. 
The factors attributable to the poor prognosis of CUA in our series are not clear. Possible reasons for the high mortality include delayed diagnosis due to low index of suspicion emanating from alternative diagnosis, including more common diabetic skin ulcer and osteomyelitis; both conditions may coexist with the calciphylaxis itself [20]. Furthermore, other skin lesions such as vasculitis may mimic calciphylaxis clinically including painful nonhealing ulcers due to ischemia which might have led to delayed diagnosis. However, with the high index of suspicion of CUA in our cohort makes it less likely as a contributing factor. On the other hand, site of CUA lesion has been reported to have prognostic value. Some authors believe proximal localization of the lesion to have worse prognosis $[1,21]$. Interestingly, only 2 of our subjects had proximal involvement yet $83 \%$ of them died despite multi-interventional approach; in agreement with others findings $[1,2]$. Another confounding prognostic factor is comorbidities. Five of the 6 subjects studied had at least 3 other diseases apart from the CUA to cope with. Furthermore, the cause of the calciphylaxis may have prognostic significance. Nonuremic calciphylaxis tends to have a less ominous course compared to those with ESRF $[3,4]$ in support of high mortality in our study in which all the subjects had renal failure from various aetiologies on haemodialysis.

The major limitation of our study was small sample size which limits power to detect associations reflecting relative rarity of this condition in clinical practice. Furthermore, the biochemistry profile presented was limited to at the time of diagnosis and within 6 months after and did not address daily changes which may have occurred in the course of followup of the subjects. Nonuniformity of treatment of calciphylaxis also makes it hard to conclude outcome as poor as reported. Furthermore, contribution of other risk factors of ischemic heart disease was not assessed in spite of myocardial infarction being one of the common causes of death in the study population. Lastly, it is important to note that there is a limitation to retrospective studies in general. Observations derived from such studies may contain some missing information and thus may serve as a stimulus to further prospective work to clarify findings. The present work must be interpreted in the knowledge of the defects inherent in such studies. Despite these, our result is consistent with other reports $[2,10]$. The study has further suggested calciphylaxis, a syndrome with high mortality irrespective of mode of care.

In conclusion, prognosis of CUA in subjects with ESRF remains poor in spite of combination therapy and multimodality of wound care. Further prospective studies on a larger population are needed to characterise our findings.

\section{References}

[1] R. H. Weenig, L. D. Sewell, M. D. P. Davis, J. T. McCarthy, and M. R. Pittelkow, "Calciphylaxis: natural history, risk factor analysis, and outcome," Journal of the American Academy of Dermatology, vol. 56, no. 4, pp. 569-579, 2007.

[2] A. R. Mazhar, R. J. Johnson, D. Gillen et al., "Risk factors and mortality associated with calciphylaxis in end-stage renal disease," Kidney International, vol. 60, no. 1, pp. 324-332, 2001.

[3] S. U. Nigwekar, M. Wolf, R. H. Sterns, and J. K. Hix, "Calciphylaxis from nonuremic causes: a systematic review," Clinical Journal of the American Society of Nephrology, vol. 3, no. 4, pp. 1139-1143, 2008.

[4] U. H. Malabu, L. J. Roberts, and K. S. Sangla, "Calciphylaxis in a morbidly obese female with rheumatoid arthritis presenting with severe weight Loss and vitamin D deficiency," Endocrine Practice, pp. 1-12, 2011.

[5] A. R. Sood, L. D. Wazny, C. B. Raymond et al., "Sodium thiosulfate-based treatment in calcific uremic arteriolopathy: a consecutive case series," Clinical Nephrology, vol. 75, no. 1, pp. 8-15, 2011.

[6] M. R. Hayden and D. J. A. Goldsmith, "Sodium thiosulfate: new hope for the treatment of calciphylaxis," Seminars in Dialysis, vol. 23, no. 3, pp. 258-262, 2010.

[7] C. Basile, A. Montanaro, M. Masi, G. Pati, P. De Maio, and A. Gismondi, "Hyperbaric oxygen therapy for calcific uremic arteriolopathy: a case series," Journal of Nephrology, vol. 15, no. 6, pp. 676-680, 2002.

[8] I. A. Mohammed, V. Sekar, A. J. Bubtana, S. Mitra, and A. J. Hutchison, "Proximal calciphylaxis treated with calcimimetic “Cinacalcet”, Nephrology Dialysis Transplantation, vol. 23, no. 1, pp. 387-389, 2008.

[9] J. Bishop, E. Brown, A. Podesta, C. Troy, and X. E. Dong, "Surgical management of calciphylaxis associated with primary hyperparathyroidism: a case report and review of the literature," International Journal of Endocrinology, vol. 2010, 4 pages, 2010.

[10] I. Kyritsis, A. Gombou, I. Griveas, I. Agroyannis, K. Retsa, and B. Agroyannis, "Combination of sodium thiosulphate, cinacalcet, and paricalcitol in the treatment of calciphylaxis with hyperparathyroidism," International Journal of Artificial Organs, vol. 31, no. 8, pp. 742-744, 2008.

[11] M. D. Arenas, M. T. Gil, M. D. Gutiérrez et al., "Management of calcific uremic arteriolopathy (calciphylaxis) with a combination of treatments, including hyperbaric oxygen therapy," Clinical Nephrology, vol. 70, no. 3, pp. 261-264, 2008.

[12] C. Baldwin, M. Farah, M. Leung et al., "Multi-intervention management of calciphylaxis: a report of 7 cases," American Journal of Kidney Diseases, vol. 58, no. 6, pp. 988-991, 2011.

[13] E. A. Ross, "Evolution of treatment strategies for calciphylaxis," American Journal of Nephrology, vol. 34, no. 5, pp. 460-467, 2011.

[14] S. Schliep, G. Schuler, and F. Kiesewetter, "Successful treatment of calciphylaxis with pamidronate," European Journal of Dermatology, vol. 18, no. 5, pp. 554-556, 2008.

[15] D. Kakagia, P. Kriki, E. Thodis, A. Roumeliotis, and V. Vargemezis, "Calcific uremic arteriolopathy treated with cinacalcet, paricalcitol, and autologous growth factors," Journal of Cutaneous Medicine and Surgery, vol. 15, no. 2, pp. 121-124, 2011.

[16] H. Maeda, M. Tokumoto, H. Yotsueda et al., "Two cases of calciphylaxis treated by parathyroidectomy: importance of increased bone formation," Clinical Nephrology, vol. 67, no. 6, pp. 397-402, 2007.

[17] N. Alikadic, D. Kovac, M. Krasna et al., "Review of calciphylaxis and treatment of a severe case after kidney transplantation with iloprost in combination with hyperbaric oxygen and cultured autologous fibrin-based skin substitutes," Clinical Transplantation, vol. 23, no. 6, pp. 968-974, 2009.

[18] L. Noureddine, M. Landis, N. Patel, and S. M. Moe, "Efficacy of sodium thiosulfate for the treatment for calciphylaxis," Clinical Nephrology, vol. 75, no. 2, pp. 485-490, 2011. 
[19] B. R. Don and A. I. Chin, "A strategy for the treatment of calcific uremic arteriolopathy (calciphylaxis) employing a combination of therapies," Clinical Nephrology, vol. 59, no. 6, pp. 463-470, 2003.

[20] S. U. Nigwekar, "An unusual case of nonhealing leg ulcer in a diabetic patient," Southern Medical Journal, vol. 100, no. 8, pp. 851-852, 2007.

[21] F. Llach, "The evolving clinical features of calciphylaxis," Kidney International, Supplement, vol. 63, no. 85, pp. S122-S124, 2003. 


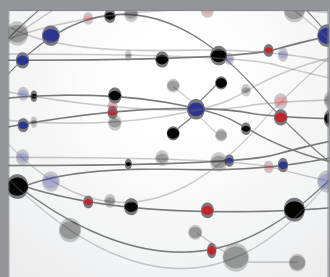

The Scientific World Journal
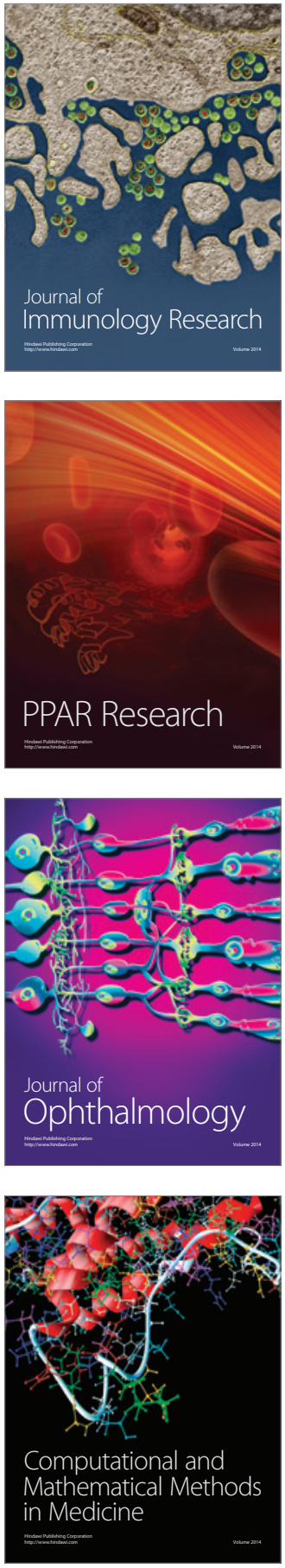

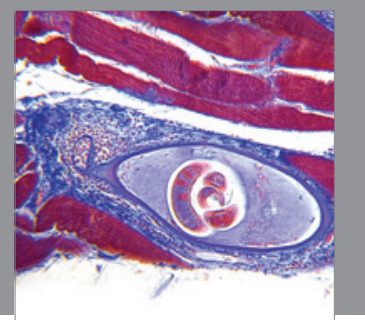

Gastroenterology

Research and Practice
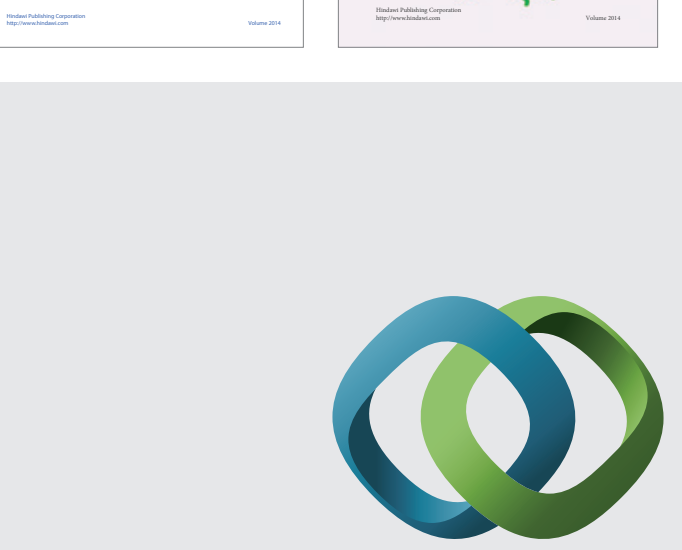

\section{Hindawi}

Submit your manuscripts at

http://www.hindawi.com
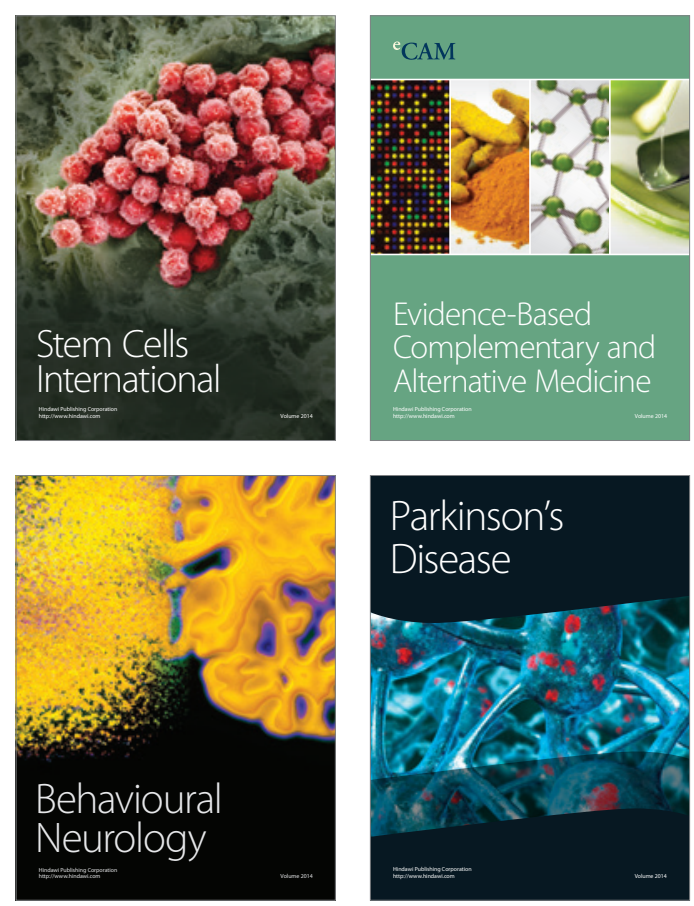

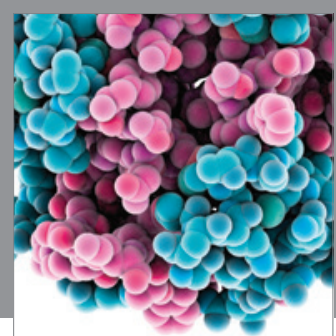

Journal of
Diabetes Research

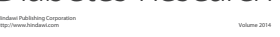

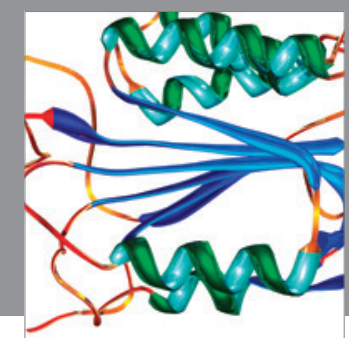

Disease Markers
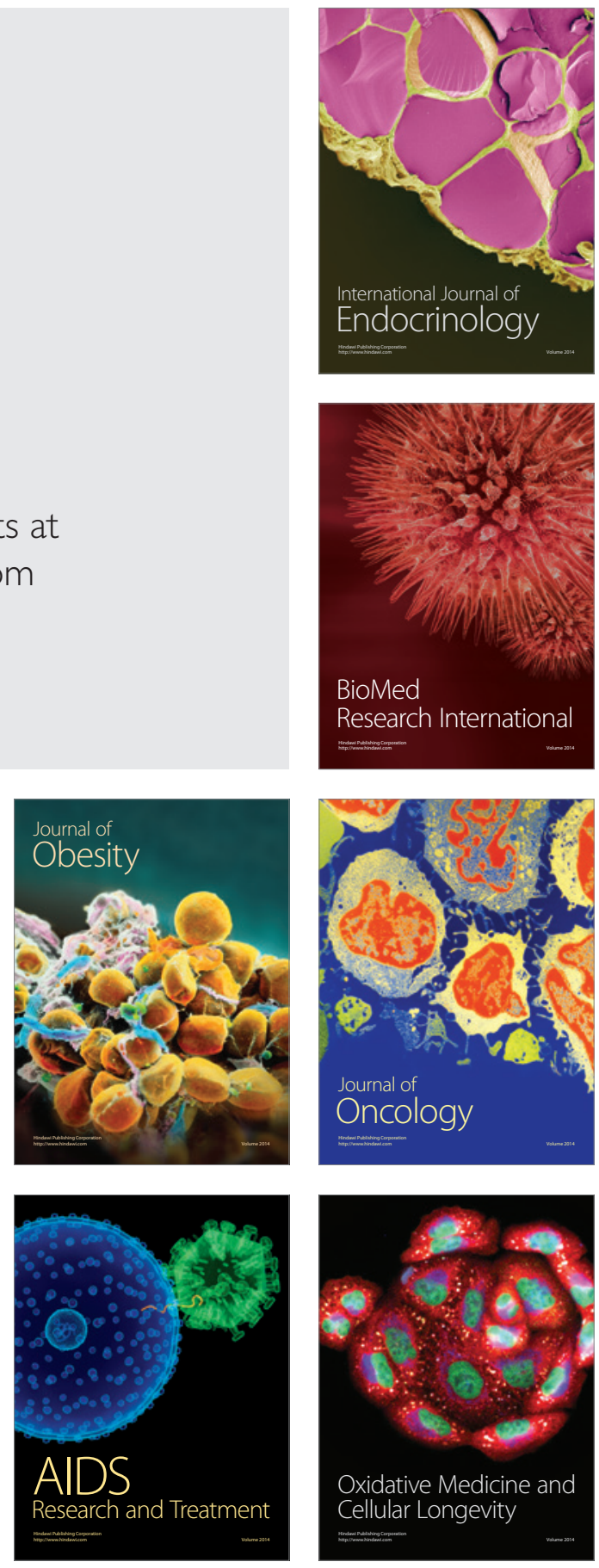